\title{
Power-Efficient VLC Transmitter Able to Reproduce Multi-Carrier Modulation Schemes by Using the Output Voltage Ripple of the HB-LED Driver
}

\author{
Juan Rodríguez ${ }^{1}$, Diego G. Lamar ${ }^{1}$, Daniel G. Aller ${ }^{1}$, Pablo F. Miaja ${ }^{2}$, and Javier Sebastián ${ }^{1}$. \\ Departamento de Ingeniería Eléctrica, Electrónica, de Computadores y Sistemas, Universidad de Oviedo, Gijón 33204, \\ Spain (e-mail: rodriguezmjuan@uniovi.es)
}

Power System Division, European Space Agency, Noordwijk 2201 AZ, The Netherlands (e-mail: pablo.fernandez@esa.int)

\begin{abstract}
Visible Light Communication (VLC) consists in using High-Brightness LEDs (HB-LEDs) not only for lighting, but also for transmitting information by changing the light intensity rapidly. One of the most important problems of VLC is that the HB-LED drivers that have been proposed for achieving the highest bit rates offer low power efficiency. The reason is that since these HB-LED drivers must be able to reproduce fast current waveforms, the use of Linear Power Amplifiers (LPAs) has been adopted, which damages the power efficiency of HB-LED lighting. To the author's knowledge, there is only one approach reported in the literature that is able to reproduce the most recommended modulation schemes by using a power efficient HB-LED driver. However, since it is based on the use of pulse-width modulated dc-dc power converters, the required switching frequency is between 3 and 6 times higher than the maximum frequency of the communication signal. As a result, the approach suffers from high switching losses when the highest bandwidths are addressed. A method for reproducing the most recommended modulation schemes by modulating both the amplitude and the phase of the output voltage ripple is presented in this work. The major benefits are the high efficiency, the simplicity and the lower switching frequency required for the implementation.
\end{abstract}

Keywords-Visible Light Communication (VLC), HighBrightness LED (HB-LED), Ripple Modulation (RM) Technique, Multi-Carrier Modulation (MCM).

\section{INTRODUCTION}

Nowadays, wireless communication is essential for the society, enabling a lot of new applications that have recently emerged based on wireless connectivity. As a result, the mobile data traffic has grown exponentially during last decade, which has been translated into a saturation of the Radio Frequency (RF) spectrum [1]. Visible Light Communication (VLC) is one of the advanced candidates for alleviating the congestion of the RF spectrum [2]. It consists in using High-Brightness LEDs (HB-LEDs) not only for lighting, but also for transmitting information by changing the light intensity rapidly. In this application, the light intensity $[\mathrm{s}(\mathrm{t})]$ has a DC component $\left(\mathrm{s}_{\mathrm{DC}}\right)$ that determines the lighting level and an $\mathrm{AC}$ component $\left[\mathrm{s}_{\mathrm{AC}}(\mathrm{t})\right]$ that represents the transmitted information:

$$
s(t)=s_{D C}+s_{A C}(t) .
$$

It is important to note that for safety reasons, the frequency components of $s_{A C}(t)$ are high enough to be unappreciable to the human eye (i.e. above $100 \mathrm{~Hz}$ approximately [3]).

Depending on the kind of HB-LED, its bandwidth ranges between 3 and $20 \mathrm{MHz}$ [4]. However, the bit rate depends not

only on the available bandwidth, but also on the modulation scheme used to transmit the information. There are three main modulation strategies for VLC: Pulse-Based Modulation (PBM) schemes, Single-Carrier Modulation (SCM) schemes and Multi-Carrier Modulation (MCM) schemes. PBM consists in transmitting the information using light pulses. PBM schemes can be reproduced easily, but they are inefficient from the communication perspective and, as a result, they are not recommended for addressing high bit rates. Both SCM and MCM schemes are better options for satisfying this target [5]. In SCM schemes, the information is transmitted by changing the amplitude and the phase of a sinusoidal waveform (see Fig. 1). In this case, the communication component of $s(t)$ [i.e., $s_{A C}(t)$ ] can be described as follows:

$$
\begin{aligned}
& S_{S C M}(t)= \\
& \quad=A_{S-S C M}(t) \cdot \cos \left(2 \cdot \pi \cdot f_{S C M} \cdot t+\phi_{S-S C M}(t)\right),
\end{aligned}
$$

where $\mathrm{f}_{\mathrm{SCM}}, \mathrm{A}_{\mathrm{S}-\mathrm{SCM}}(\mathrm{t})$ and $\phi_{\mathrm{S}-\mathrm{SCM}}(\mathrm{t})$ are the frequency of the sinusoid, the amplitude modulation and the phase modulation, respectively. Moreover, MCM schemes are made up of $N$ sinusoidal waveforms with different frequencies $\left(\mathrm{f}_{\mathrm{MCM}-1}\right.$, $\left.\mathrm{f}_{\mathrm{MCM}-2}, \ldots \mathrm{f}_{\mathrm{MCM}-\mathrm{N}}\right)$ that also change the amplitude and the phase over time (see Fig. 2). In this case, $\mathrm{s}_{\mathrm{AC}}(\mathrm{t})$ can be expressed as follows:

$$
\begin{aligned}
& s_{M C M}(t)=\frac{1}{N} \sum_{k=1}^{N} s_{M C M-k}(t), \\
& s_{M C M-k}(t)=A_{S-M C M-k}(t) \cdot \\
& \cdot \cos \left(2 \cdot \pi \cdot f_{M C M-k} \cdot t+\phi_{S-M C M-k}(t)\right) .
\end{aligned}
$$

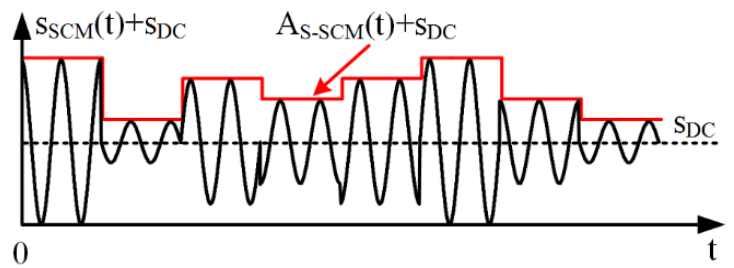

0

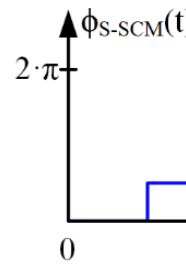

Fig. 1. Example of a SCM scheme for VLC: (a) SCM signal highlighting the amplitude. (b) Phase of the SCM signal. 


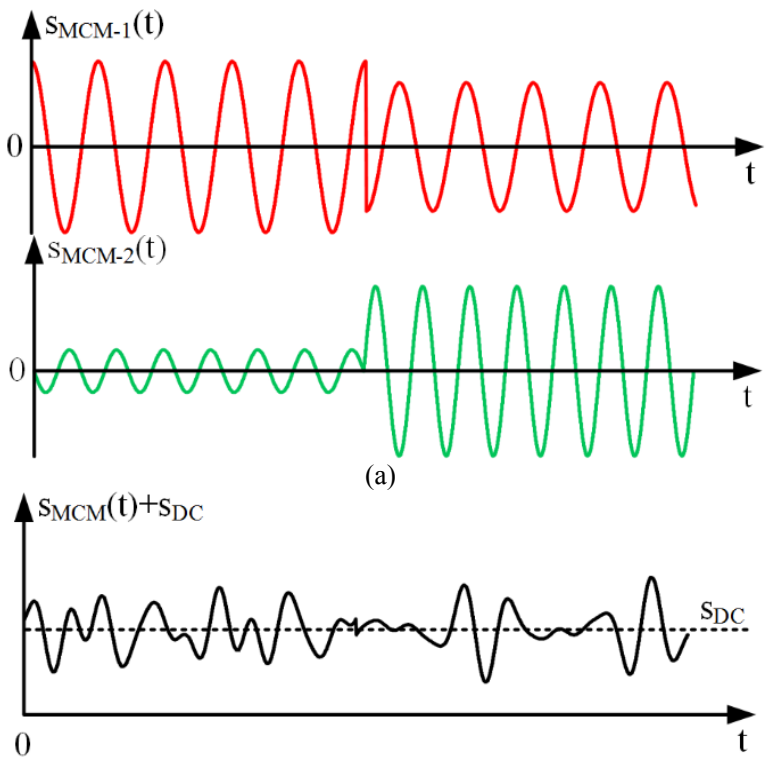

(b)

Fig. 2. Example of a MCM scheme for VLC: (a) Two of the sinusoids of the MCM schemes. (b) MCM signal.

Besides the bit rate, achieving low error during the demodulation process is essential for reaching a successful communication. In wireless communication, this error is strongly jeopardized by the Multipath Fading (MF). This phenomenon is caused by the different propagation paths of the signal, which is translated into different time delays of the light beams that reach the receiver, increasing the error during the demodulation process. Differently from SCM schemes, MCM schemes are robust against the MF problem and, as a consequence, they are the preferred modulation strategy for VLC [6]. The problem is that MCM schemes are reproduced by using Class A or AB Linear Power Amplifiers (LPAs), which offer a power efficiency that ranges between $10 \%$ and $40 \%$ [2]. Therefore, these LPAs jeopardize one of the main advantages of HB-LED lighting: the high power efficiency. This work aims to introduce a method for reproducing MCM schemes by using the output voltage ripple of a power converter, thus keeping high power efficiency. The paper is organized as follows. The operating principle of the HB-LED driver proposed for VLC is explained in Section II. The control system is described in Section III. The experimental results are given in Section IV and, finally, the conclusions are gathered in Section V.

\section{REPRODUCING MCM SCHEMES BY USING THE OUTPUT VOLTAGE RIPPlE OF A POWER CONVERTER}

\section{A. HB-LED Drivers able to Reproduce MCM Schemes}

Using a HB-LED driver fully based-on the use of power converters is a promising approach for avoiding the efficiency problem of LPAs [7]-[8]. However, to the author's knowledge, only a HB-LED driver for VLC able to reproduce MCM schemes can be found in the literature. The HB-LED driver presented in [9] is based on the use of pulse-width modulated dc-dc power converters, and is able to reproduce both SCM and MCM schemes. As Fig. 3(a) shows, the drawback of this approach is that the Pulse-Width Modulation (PWM) technique requires a switching frequency ( $\mathrm{f}_{\mathrm{SW}-\mathrm{PWM}}$ ) around 20 times higher than the maximum frequency of the communication signal ( $\left.f_{S-M a x}\right)$ [10]. As a result, switching losses are unaffordable when the highest bandwidths are addressed and both the devices of the power stage and the digital system responsible for the gate signals generation are

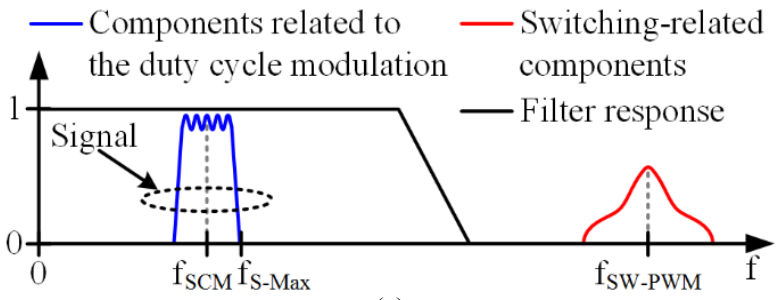

(a)

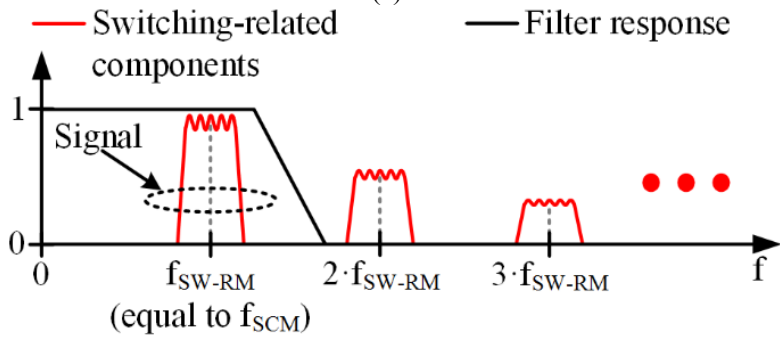

(b)

Fig. 3. Comparison of the approaches based on the use of power converters in the frequency domain: (a) PWM technique. (b) RM technique.

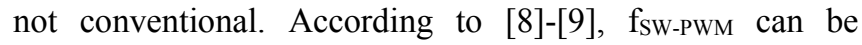
reduced to around 3 or 6 times $f_{\mathrm{S}-\mathrm{Max}}$ by using sophisticated buck-derived converters.

A technique that enables the use of a lower switching frequency is reported in [11]. This technique will be noted as Ripple Modulation (RM) and it consists in modulating both the amplitude and the phase of the first switching harmonic of the output voltage ripple in order to reproduce an SCM signal. It is important to note that in the case of the RM technique, the switching frequency ( $f_{\mathrm{SW}-\mathrm{RM}}$ ) is equal to the frequency of the $\mathrm{SCM}$ sinusoid $\left(\mathrm{f}_{\mathrm{SCM}}\right)$. As a result, $\mathrm{f}_{\mathrm{SW}-\mathrm{RM}}$ is much lower than $\mathrm{f}_{\mathrm{SW}-\mathrm{PWM}}$ (see Fig. 3), which is translated into a high reduction of the switching losses and a less challenging implementation. For instance, if $\mathrm{f}_{\mathrm{SCM}}$ is $10 \mathrm{MHz}$ and $\mathrm{f}_{\mathrm{S}-\mathrm{Max}}$ is $13 \mathrm{MHz}, \mathrm{f}_{\mathrm{SW}-\mathrm{PWM}}$ would be around $60 \mathrm{MHz}$ whereas $\mathrm{f}_{\mathrm{SW}-\mathrm{RM}}$ would be $10 \mathrm{MHz}$. The main drawback of the RM technique is that it has not been reported how to reproduce MCM schemes. This work aims to address this point and, moreover, to clearly describe the control system of the power converter.

\section{B. Operating Principle of the RM Technique}

As was previously mentioned, the RM technique enables the reproduction of SCM signals by modulating both the amplitude and the phase of the first switching harmonic of the output voltage ripple [11]. The HB-LED driver topology proposed for applying this technique is a two-phase synchronous buck converter with $M^{\text {th }}$ order output filter (see Fig. 4). Differently from a conventional buck converter, the output filter passes not only the DC component, but also the first-switching harmonic of the pulse voltage waveforms of the switch-nodes [v-A $(\mathrm{t})$ and $\left.\mathrm{v}_{\mathrm{S}-\mathrm{B}}(\mathrm{t})\right]$. Therefore, and as Fig. 3 (b) shows, the cut-off frequency of the filter $\left(\mathrm{f}_{\mathrm{C}}\right)$ must be higher than the first switching harmonic and lower than the second one (i.e., $\mathrm{f}_{\mathrm{SW}-\mathrm{RM}}<\mathrm{f}_{\mathrm{C}}<2 \cdot \mathrm{f}_{\mathrm{SW}-\mathrm{RM}}$ ). In this way, the output voltage $\left[\mathrm{vO}_{\mathrm{O}}(\mathrm{t})\right]$ is made up of a DC component ( $\mathrm{V}_{\mathrm{O}-\mathrm{DC}}$ ) and a sinusoid $\left[\mathrm{V}_{\mathrm{O}-\mathrm{AC}}(\mathrm{t})\right]$ whose frequency is $\mathrm{f}_{\mathrm{SW}-\mathrm{R}}$ :

$$
\begin{gathered}
v_{O}(t)=v_{O-D C}+v_{O-A C}(t), \\
v_{O-D C}=d \cdot V_{G}, \\
v_{O-A C}(t)=A_{V}(t) \cdot \cos \left(2 \cdot \pi \cdot f_{S W-R M} \cdot t+\phi_{V}(t)\right),
\end{gathered}
$$

where $d$ is the duty cycle, $V_{G}$ is the input voltage, $A_{V}(t)$ is the amplitude of the sinusoid and $\phi_{\mathrm{V}}(\mathrm{t})$ is the phase of the sinusoid. 


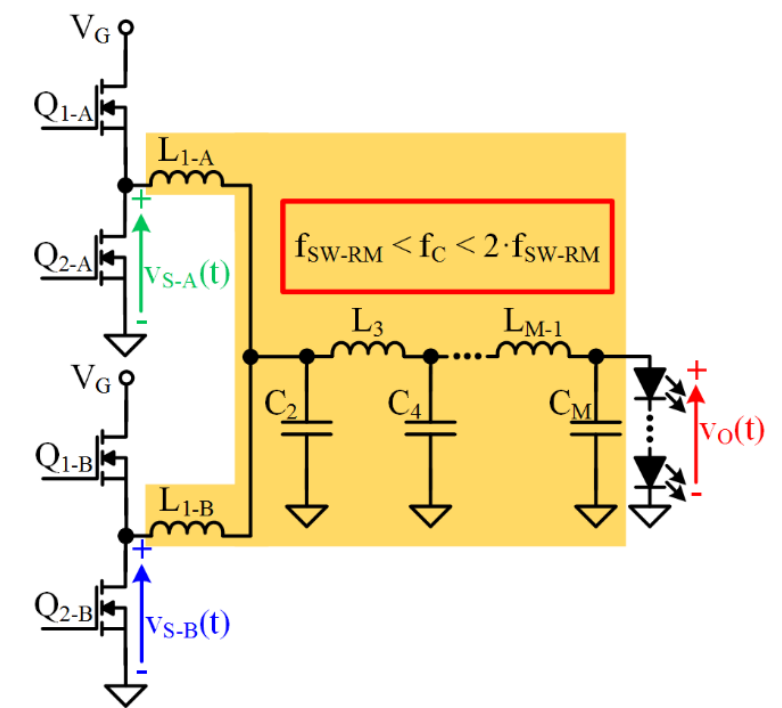

Fig. 4. Two-phase synchronous buck converter with no conventional filter for applying the RM technique.

Differently from the PWM technique, the RM technique is based on controlling not only the width of the voltage pulses at the input of the filter [i.e., the duty cycle of vs-A $(t)$ and $\left.\mathrm{v}_{\mathrm{S}-\mathrm{B}}(\mathrm{t})\right]$, but also the position over the switching period (see Fig. 5). In particular, the width modulation is the same for both phases, but the position over the switching period is controlled individually for each one. As will be demonstrated later, this strategy enables the control of both $A_{V}(t)$ and $\phi_{V}(t)$. It is important to note that controlling the position of the pulses is equivalent to control their phases. The dimensionless parameter that is used to control the phase of a pulse will be noted as $\gamma$ [see Fig. 5 (b)], and it ranges between 0 and 1 . When $\gamma$ is 0 , the phase is $0^{\circ}$, and when $\gamma$ is 1 , the phase is $-2 \cdot \pi$. Therefore, the phase of a pulse is equal to $-\gamma \cdot 2 \cdot \pi$ and, as a consequence, the center of the pulse appears $\gamma \cdot \mathrm{T}_{\mathrm{SW}}$ after the beginning of the switching period [see Fig. 5 (b)]. Note that the phase is determined by the center of the pulse.

The equivalent circuits depicted in Fig. 6 will support the explanation of how $A_{V}(t)$ and $\phi_{V}(t)$ can be modulated by controlling the phase (i.e., the position) of vs-A $(\mathrm{t})$ and $\mathrm{v}_{\mathrm{S}-\mathrm{B}}(\mathrm{t})$. Basically, $v_{S-A}(t)$ and $v_{S-B}(t)$ are pulse voltage waveforms determined by the MOSFETs $\left[\mathrm{Q}_{1-\mathrm{A}}\right.$ and $\mathrm{Q}_{2-\mathrm{A}}$ in the case of $\mathrm{V}_{\mathrm{S}-\mathrm{A}}(\mathrm{t})$, and $\mathrm{Q}_{1-\mathrm{B}}$ and $\mathrm{Q}_{2-\mathrm{B}}$ in the case of $\left.\mathrm{V}_{\mathrm{S}-\mathrm{B}}(\mathrm{t})\right]$. Therefore, these voltages can be considered as ideal pulse voltage sources [see Fig. 6(a)]. The superposition theorem allows us to calculate $v_{0}(t)$ by analyzing the voltage contribution of each phase [see Fig. 6(b)]:

$$
v_{O}(t)=v_{O-A}(t)+v_{O-B}(t)
$$

where $\mathrm{v}_{\mathrm{O}-\mathrm{A}}(\mathrm{t})$ and $\mathrm{v}_{\mathrm{O}-\mathrm{B}}(\mathrm{t})$ are the voltages provided by phases A and B, respectively. The circuit depicted in Fig. 6(c) can be obtained by considering that the phase inductors are equal and by applying the Thevenin's theorem. This circuit considers $\mathrm{VS}_{\mathrm{A}} \mathrm{A}(\mathrm{t})$ divided by two and applied to a low-pass filter, where the equivalent first inductor $\left(\mathrm{L}_{1}\right)$ is half the phase inductor. Taking into account that the filter passes not only the DC component, but also the first switching harmonic; the voltage contribution of each phase can be calculated as follows:

$$
\begin{gathered}
v_{O-A}(t)=v_{O-A-D C}+v_{O-A-A C}(t), \\
v_{O-A-D C}=\frac{d \cdot V_{G}}{2}
\end{gathered}
$$

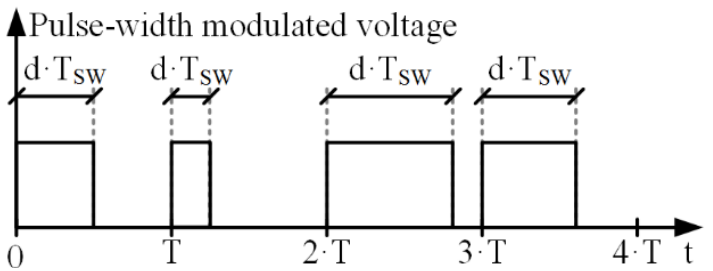

(a)

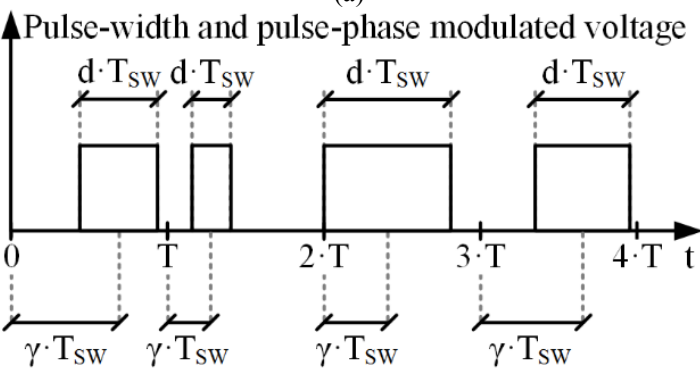

(b)

Fig. 5. Two strategies for controlling a power converter: (a) Pulse-width modulation control. (b) Pulse-width and pulse-phase modulation control.

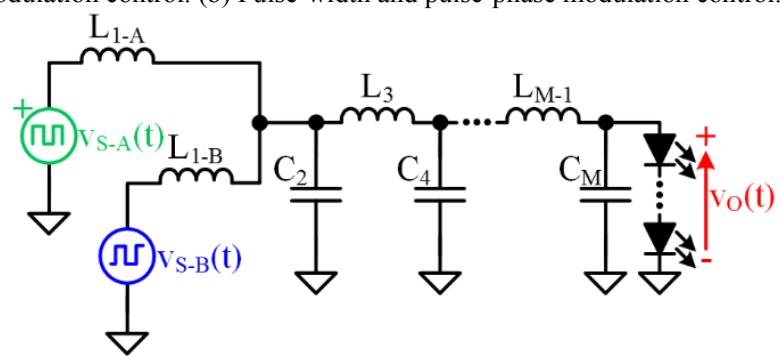

(a)

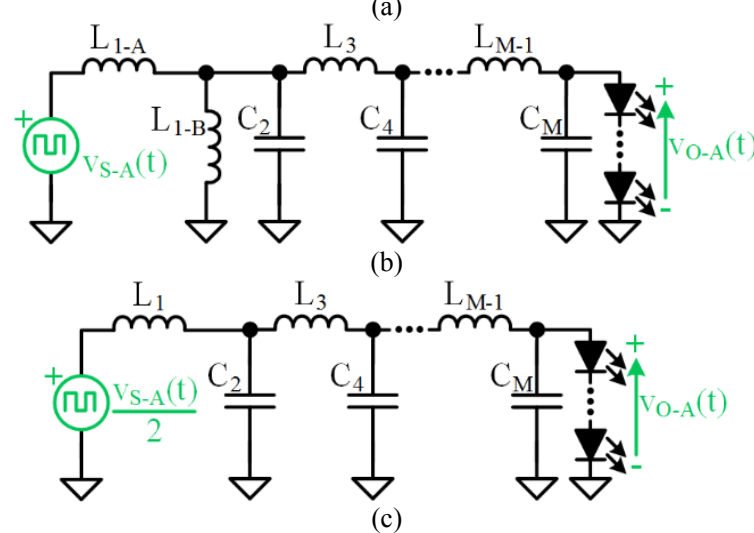

Fig. 6. Equivalent circuits of the two-phase synchronous buck converter for applying the RM technique: (a) Considering the switch-node voltages as ideal pulse voltage sources. (b) Applying the superposition theorem (example with phase A). (c) Applying the Thevenin's theorem (example with phase A).

$$
\begin{gathered}
v_{O-A-A C}(t)=\frac{V_{G}}{\pi} \sin (d \cdot \pi) \cdot \\
\cdot \cos \left(2 \cdot \pi \cdot f_{S W-R M} \cdot t-\gamma_{A}(t) \cdot 2 \cdot \pi\right), \\
v_{O-B}(t)=v_{O-B-D C}+v_{O-B-A C}(t), \\
v_{O-B-D C}=\frac{d \cdot V_{G}}{2}, \\
v_{O-B-A C}(t)=\frac{V_{G}}{\pi} \sin (d \cdot \pi) \cdot \\
\cdot \cos \left(2 \cdot \pi \cdot f_{S W-R M} \cdot t-\gamma_{B}(t) \cdot 2 \cdot \pi\right),
\end{gathered}
$$

where $\gamma_{\mathrm{A}}(\mathrm{t})$ and $\gamma_{\mathrm{B}}(\mathrm{t})$ are the dimensionless control parameters that determine the phase of the voltage pulses (i.e., the position of the pulses over the switching period) [see Fig. 7(a)-(b)]. Substituting (9)-(14) into (8) allows us to obtain $\mathrm{vo}_{\mathrm{O}}(\mathrm{t})$ : 


$$
\begin{gathered}
v_{O-D C}=v_{O-A-D C}+v_{O-B-D C}=d \cdot V_{G}, \\
v_{O-A C}(t)=v_{O-A-A C}(t)+v_{O-B-A C}(t)= \\
=A_{V}\left[\gamma_{1}(t), \gamma_{2}(t), d\right] \cdot \\
\cdot \cos \left(2 \cdot \pi \cdot f_{S W-R M} \cdot t+\phi_{V}\left[\gamma_{1}(t), \gamma_{2}(t)\right]\right), \\
A_{V}\left[\gamma_{1}(t), \gamma_{2}(t), d\right]= \\
=\frac{2 \cdot V_{G}}{\pi} \sin (d \cdot \pi) \cos \left[\left(\gamma_{2}(t)-\gamma_{1}(t)\right) \cdot \pi\right], \\
\phi_{V}\left[\gamma_{1}(t), \gamma_{2}(t)\right]=\frac{\gamma_{1}(t)+\gamma_{2}(t)}{2} \cdot 2 \cdot \pi .
\end{gathered}
$$

Therefore, the phases of the pulses determine both $A_{V}(t)$ and $\phi_{V}(t)$. In particular, $A_{V}(t)$ depends on the phase-shift between the pulses, and $\phi_{\mathrm{V}}(\mathrm{t})$ depends on the average phase of the pulses [see Fig. 7(c)]. Hence, it is recommendable to focus on the phase-shift and on the average phase instead of on the individual phases. In this way, two new parameter are introduced in order to replace $\gamma_{1}(t)$ and $\gamma_{2}(t)$. First, $\alpha(t)$ is an dimensionless parameter that determines the phase-shift between the pulses (i.e., the position difference) and, as a consequence, $A_{V}(t)$. Second, $\beta(t)$ determines the average phase of the pulses (i.e., the average position) and, as a result, $\phi_{\mathrm{V}}(\mathrm{t})$. These two parameters can be defined as follows:

$$
\begin{aligned}
& \alpha(t)=\gamma_{2}(t)-\gamma_{1}(t), \\
& \beta(t)=\frac{\gamma_{1}(t)+\gamma_{2}(t)}{2} .
\end{aligned}
$$

It is considered that $\gamma_{1}(t)$ is always lower than $\gamma_{2}(t)$ and that the difference never overcomes 0.5 . This ensures that $A_{v}(t)$ is always higher than 0 .

Substituting (19) and (20) into (17) and (18) leads to the following equations:

$$
\begin{gathered}
A_{V}[\alpha(t), d]=\frac{2 \cdot V_{G}}{\pi} \sin (d \cdot \pi) \cos [\alpha(t) \cdot \pi], \\
\phi_{V}[\beta(t)]=\beta(t) \cdot 2 \cdot \pi .
\end{gathered}
$$

In summary, the RM technique allows us to provide an output voltage that is made up of a DC component and a sinusoid whose frequency is equal to the switching frequency (i.e., $f_{\text {Sw-RM }}$ ). The duty cycle (i.e., d) determines vo-Dc. The amplitude of the sinusoid [i.e., $\left.\mathrm{Av}_{\mathrm{v}}(\mathrm{t})\right]$ and its phase [i.e., $\phi_{\mathrm{v}}(\mathrm{t})$ ] can be controlled with the phase-shift between the pulses [i.e., by using $\alpha(t)]$ and with the average phase of the pulse [i.e., by using $\beta(\mathrm{t})$ ], respectively. In order to reproduce a particular waveform, the first step is to determine $\mathrm{d}$ to obtain the desired DC component:

$$
d=\frac{v_{O-D C}}{V_{G}} .
$$

The second step is to calculate $\alpha(\mathrm{t})$ in order to obtain the desired amplitude of the output voltage sinusoid:

$$
\alpha(t)=\frac{1}{\pi} \cos ^{-1}\left(\frac{\pi \cdot A_{V}(t)}{2 \cdot V_{G} \cdot \sin (d \cdot \pi)}\right) .
$$

Finally, the third step is to calculate $\beta(t)$ in order to obtain the desired phase of the output voltage:

$$
\beta(t)=\frac{-\phi_{V}(t)}{2 \cdot \pi} .
$$

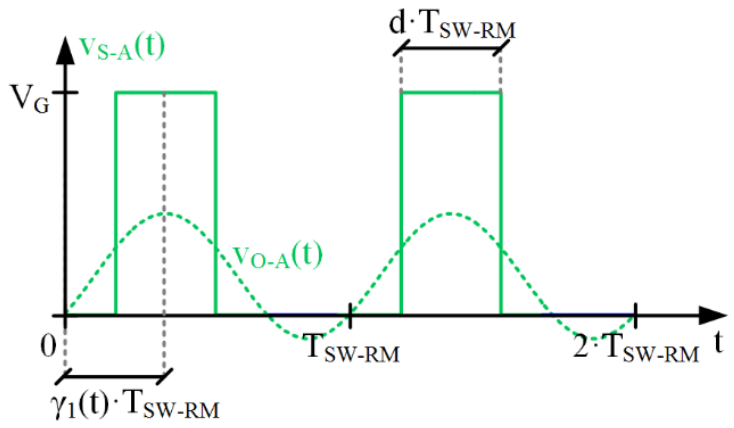

(a)

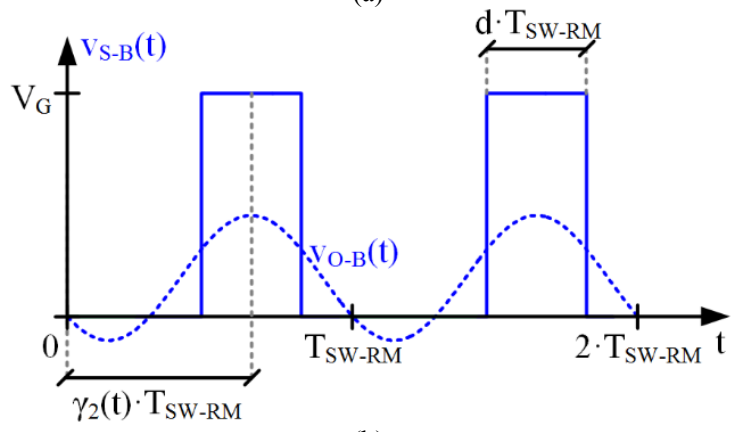

(b)

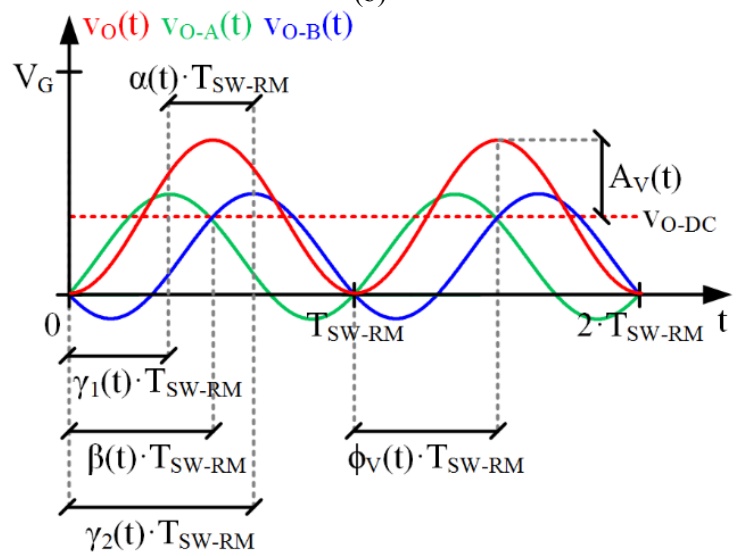

(c)

Fig. 7. Main voltage waveforms of the two-phase synchronous buck converter used to apply the RM technique: (a) Switch-node voltage of phase A and output voltage component that it provides. (b) Switch-node voltage of phase B and output voltage component that it provides. (c) Graphical calculation of the output voltage from the components provided by each phase.

\section{Using the RM Technique to Reproduce MCM Schemes}

As previously explained, the RM technique has an important drawback: it was proposed for reproducing SCM schemes and it has not been reported how to reproduce MCM schemes (i.e., the most recommended modulation schemes for VLC). Reproducing SCM schemes using the RM technique is straightforward by comparing equation (2) (i.e., the desired light intensity) and equations (16)-(18) (i.e., the $\mathrm{AC}$ voltage obtained with the RM technique) and by considering the linear relationship between $s(t)$ and $v_{0}(t)$ when the HB-LEDs are properly biased:

$$
\begin{gathered}
A_{V}(t)=K_{V-S} \cdot A_{S-S C M}(t), \\
\phi_{V}(t)=\phi_{S-S C M}(t),
\end{gathered}
$$

where $\mathrm{K}_{\mathrm{V}-\mathrm{S}}$ is the proportionality coefficient between $\mathrm{V}_{\mathrm{O}-\mathrm{AC}}(\mathrm{t})$ and $\mathrm{SO}_{\mathrm{AC}}(\mathrm{t})$. Hence, $\mathrm{fSW}_{\mathrm{SW}} \mathrm{RM}$ must be equal to $\mathrm{f}_{\mathrm{SCM}}, \mathrm{Av}_{\mathrm{V}}(\mathrm{t})$ must track a reference that is proportional to the amplitude modulation and $\phi_{\mathrm{v}}(\mathrm{t})$ must be equal to the phase modulation. Substituting (26) and (27) into (24) and (25) allows us to 
obtain how $\alpha(\mathrm{t})$ and $\beta(\mathrm{t})$ must be controlled in order to reproduce SCM schemes:

$$
\begin{gathered}
\alpha(t)=\frac{1}{\pi} \cos ^{-1}\left(\frac{\pi \cdot K_{V-S} \cdot A_{S-S C M}(t)}{2 \cdot V_{G} \cdot \sin (d \cdot \pi)}\right), \\
\beta(t)=\frac{-\phi_{S-S C M}(t)}{2 \cdot \pi} .
\end{gathered}
$$

The analysis for MCM schemes is not as trivial as in the case of SCM schemes. Since MCM schemes are made up of several sinusoids with different frequencies [check equations (3)-(4)], identifying the references that $A_{V}(t)$ and $\phi_{V}(t)$ must track is not straightforward. In order to obtain those references, equations (3)-(4) must be manipulated to reach an expression where only a single sinusoid changes the amplitude and the phase over time. The first step is to calculate the center frequency of the scheme as follows:

$$
f_{M C M}=\frac{1}{N} \sum_{k=1}^{N} f_{M C M-k} .
$$

After that, the difference between $\mathrm{f}_{\mathrm{MCM}}$ and the frequency of each sinusoid can be expressed as follows (see Fig. 8):

$$
\Delta f_{M C M-k}=f_{M C M-k}-f_{M C M} .
$$

Substituting (31) into (4) leads to:

$$
\begin{aligned}
& S_{M C M-k}(t)=A_{S-M C M-k}(t) \cdot \\
& \cdot \cos \left(2 \cdot \pi \cdot\left(f_{M C M}+\Delta f_{M C M-k}\right) \cdot t+\phi_{S-M C M-k}(t)\right) \\
& =A_{S-M C M-k}(t) \cdot \cos \left(2 \cdot \pi \cdot f_{M C M} \cdot t\right) \cdot \\
& \quad \cdot \cos \left(2 \cdot \pi \cdot \Delta f_{M C M-k} \cdot t+\phi_{S-M C M-k}(t)\right)- \\
& -A_{S-M C M-k}(t) \cdot \sin \left(2 \cdot \pi \cdot f_{M C M} \cdot t\right) \cdot \\
& \quad \cdot \sin \left(2 \cdot \pi \cdot \Delta f_{M C M-k} \cdot t+\phi_{S-M C M-k}(t)\right) .
\end{aligned}
$$

Substituting (32) into (3) leads to:

$$
\begin{aligned}
s_{M C M}(t) & =s_{M C M-I}(t) \cdot \cos \left(2 \cdot \pi \cdot f_{M C M} \cdot t\right)- \\
& -s_{M C M-Q}(t) \cdot \sin \left(2 \cdot \pi \cdot f_{M C M} \cdot t\right),
\end{aligned}
$$

where $\mathrm{S}_{\mathrm{MCM}-\mathrm{I}}(\mathrm{t})$ and $\mathrm{S}_{\mathrm{MCM}-\mathrm{Q}}(\mathrm{t})$ are the in-phase and quadrature component of $\mathrm{S}_{\mathrm{MCM}}(\mathrm{t})$ with respect to a sinusoid whose frequency is $\mathrm{f}_{\mathrm{MCM}}$. These two components are formulated as:

$$
\begin{aligned}
S_{M C M-I}(t) & =\frac{1}{N} \sum_{k=1}^{N} A_{S-M C M-k}(t) \cdot \\
\cdot & \cos \left(2 \cdot \pi \cdot \Delta f_{M C M-k} \cdot t+\phi_{S-M C M-k}(t)\right), \\
S_{M C M-Q}(t) & =\frac{1}{N} \sum_{k=1}^{N} A_{S-M C M-k}(t) \cdot \\
\cdot & \sin \left(2 \cdot \pi \cdot \Delta f_{M C M-k} \cdot t+\phi_{S-M C M-k}(t)\right) .
\end{aligned}
$$

Finally, the MCM signal can be expressed as a function of a single sinusoid that changes the amplitude and the phase over time:

$$
\begin{gathered}
S_{M C M}(t)=A_{S-M C M}(t) \cdot \\
\cdot \cos \left(2 \cdot \pi \cdot f_{M C M} t+\phi_{S-M C M}(t)\right), \\
A_{S-M C M}(t)=\sqrt{s_{M C M-I}(t)^{2}+s_{M C M-Q}(t)^{2}}, \\
\phi_{S-M C M}(t)=\tan ^{-1}\left(\frac{s_{M C M-Q}(t)}{s_{M C M-I}(t)}\right) .
\end{gathered}
$$

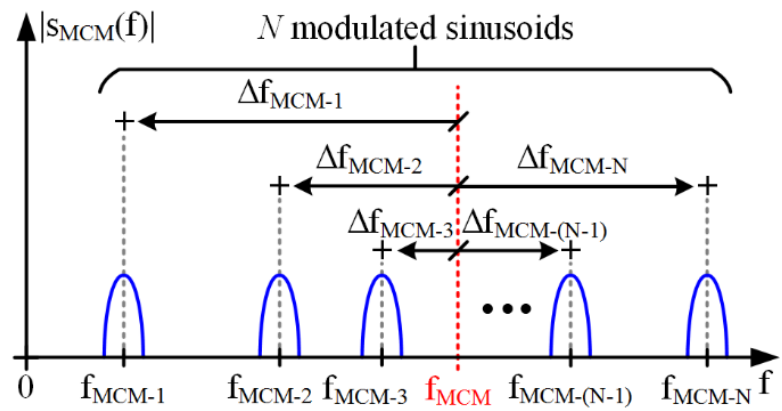

Fig. 8. MCM scheme in the frequency domain.

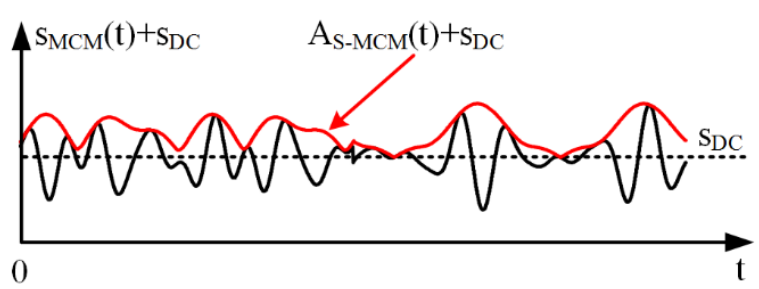

(a)

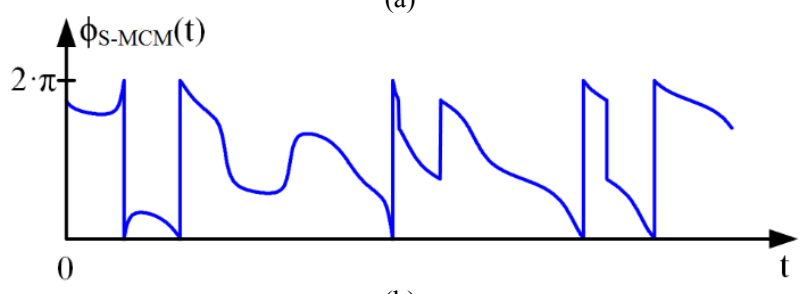

(b)

Fig. 9. Envelope (a) and instantaneous phase (b) of a MCM signal.

It is important to note that $\mathrm{AS-MCM}_{\mathrm{S}}(\mathrm{t})$ and $\phi_{\mathrm{S}-\mathrm{MCM}}(\mathrm{t})$ are the envelope and the instantaneous phase of the pass-band signal considering a sinusoid with frequency equal to $\mathrm{f}_{\mathrm{SW}-\mathrm{RM}}$ as the carrier (see Fig. 9) [5]. Therefore, the RM technique can be used to reproduce MCM schemes. In this case, $\mathrm{f}_{\mathrm{SW}-\mathrm{RM}}$ must be equal to $\mathrm{f}_{\mathrm{MCM}}, \mathrm{Av}_{\mathrm{V}}(\mathrm{t})$ must track a reference that is proportional to the envelope of the MCM signal and $\phi_{\mathrm{v}}(\mathrm{t})$ must be equal to its instantaneous phase:

$$
\begin{gathered}
A_{V}(t)=K_{V-S} \cdot A_{S-M C M}(t), \\
\phi_{V}(t)=\phi_{S-M C M}(t) .
\end{gathered}
$$

Substituting (39) and (40) into (24) and (25) allows us to obtain how $\alpha(\mathrm{t})$ and $\beta(\mathrm{t})$ must be controlled in order to reproduce $\mathrm{MCM}$ schemes:

$$
\begin{gathered}
\alpha(t)=\frac{1}{\pi} \cos ^{-1}\left(\frac{\pi \cdot K_{V-S} \cdot A_{S-M C M}(t)}{2 \cdot V_{G} \cdot \sin (d \cdot \pi)}\right) . \\
\beta(t)=\frac{-\phi_{S-M C M}(t)}{2 \cdot \pi} .
\end{gathered}
$$

\section{CONTROL SYSTEM}

As previously explained, the RM technique is based on controlling not only the width of the voltage pulses at the input of the filter [i.e., the duty cycle of $\mathrm{Vs}_{\mathrm{A}-\mathrm{A}}(\mathrm{t})$ and $\mathrm{v}_{\mathrm{S}-\mathrm{B}}(\mathrm{t})$ ], but also their phase (see Fig. 5). This fact implies that the control system of the power converter is very different from that of power converters based on the PWM technique. Fig. 10 shows the main blocks of the control system. The inputs are the voltage envelope reference $\left[A_{V-R e f}(t)\right]$, the instantaneous phase reference $\left[\phi_{\mathrm{V}-\mathrm{Ref}}(\mathrm{t})\right]$, the measurement of the current that flows through the HB-LEDs [i $\left.\mathrm{i}_{\mathrm{O}}(\mathrm{t})\right]$ and the DC current reference [i $\left.\mathrm{i}_{\mathrm{O}-\mathrm{DC}-\mathrm{Ref}}\right]$, which, in turns, determines the desired lighting level. The outputs are the pulse-voltage waveforms 


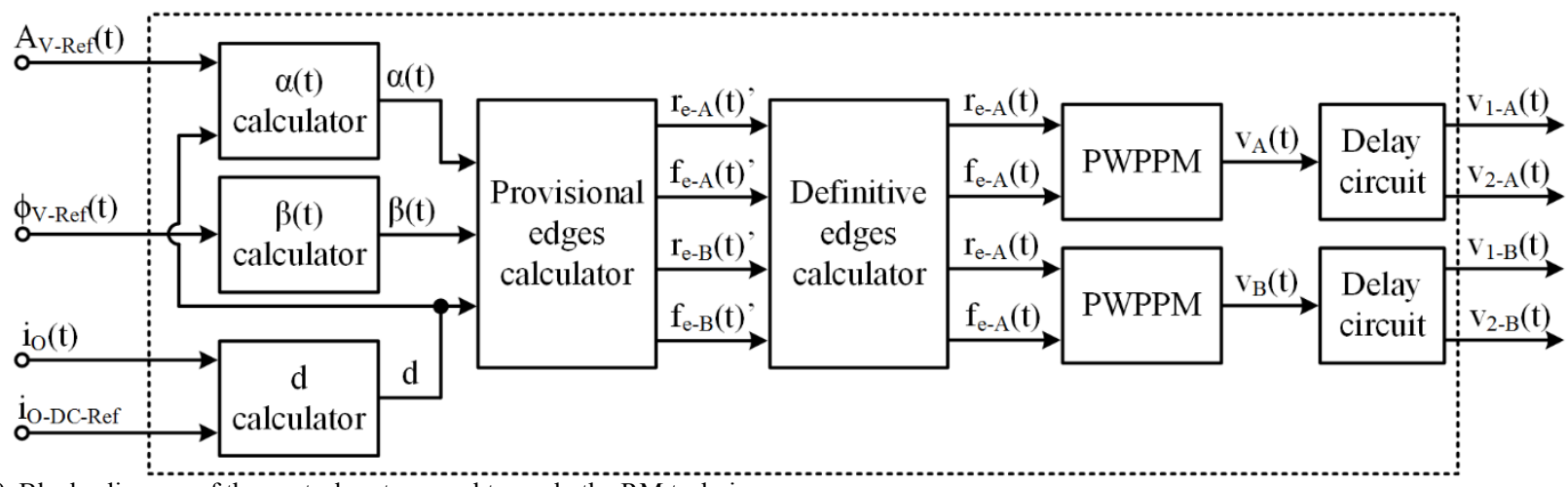

Fig. 10. Blocks diagram of the control system used to apply the RM technique.

that feed the drivers of the MOSFETs: $v_{1-A}(t), v_{2-A}(t), v_{1-B}(t)$ and $\mathrm{v}_{2-\mathrm{B}}(\mathrm{t})$ for the drivers of $\mathrm{Q}_{1-\mathrm{A}}, \mathrm{Q}_{2-\mathrm{A}}, \mathrm{Q}_{1-\mathrm{B}}$ and $\mathrm{Q}_{2-\mathrm{B}}$, respectively. A general description of the main blocks can be found below.

\section{A. Duty Cycle Calculator}

This block calculates the $\mathrm{d}$ value that is required for properly biasing the HB-LEDs (see Fig. 11). $\mathrm{i}_{\mathrm{O}}(\mathrm{t})$ is sensed

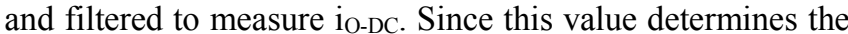
lighting level, iO-DC-Ref is compared to iO-DC. After that, the EA provides the $d$ value that ensures the desired lighting level.

\section{B. $\alpha(t)$ Calculator}

As (24) shows, $\alpha(t)$ depends not only on $A_{V}(t)$, but also on the current $d$ value. As a consequence, both $A_{v}(t)$ and $d$ feed the $\alpha(\mathrm{t})$ calculator block (see Fig. 10). According to (24), the calculation that must be carried out is not trivial. Fortunately, it can be simplified since, in practice, $A_{v}(t)$ is much smaller than $\mathrm{V}_{\mathrm{G}}$. This is because of the relationship between the light intensity emitted by an HB-LED, the current that flows through it and the applied voltage: once the HB-LED is properly biased, small voltage changes lead to both big current changes and big light intensity changes [9]. As Fig. 12 shows, the relationship between $\alpha(\mathrm{t})$ and $\mathrm{A}_{\mathrm{V}}(\mathrm{t})$ is almost linear when $A_{V}(t)<<V_{G}$ and, as a consequence, the following approximation is quite accurate:

$$
\alpha(t) \cong \alpha_{O}-m(d) \cdot A_{V}(t) .
$$

Note that $\alpha_{O}$ is the $\alpha(t)$ value when $A_{V}(t)$ is $0 \mathrm{~V}$, and $m_{O}$ is the derivate of $\alpha(t)$ with respect to $A_{V}(t)$ and evaluated in $\mathrm{A}_{\mathrm{v}}(\mathrm{t})=0 \mathrm{~V}$ :

$$
\begin{gathered}
\alpha_{O}=0.5, \\
m_{O}(d)=\frac{1}{2 \cdot V_{G} \cdot \sin (d \cdot \pi)} .
\end{gathered}
$$

Since $\mathrm{m}_{\mathrm{O}}$ depends on $\mathrm{d}$ and the function is not trivial, it is calculated by means of a look-up table (see Fig. 13).

\section{C. $\beta(t)$ Calculator}

This block is straightforward because it must multiply $\phi_{\mathrm{V}-\operatorname{Ref}}(\mathrm{t})$ by $-1 / 2 \cdot \pi$ according to $(25)$.

\section{Provisional Edges Calculator}

Once $\alpha(\mathrm{t}), \beta(\mathrm{t})$ and $\mathrm{d}$ are calculated, the generation of the pulses can be addressed. In the particular case of the twophase buck converter, both $\mathrm{v}_{\mathrm{S}-\mathrm{A}}(\mathrm{t})$ and $\mathrm{v}_{\mathrm{S}-\mathrm{B}}(\mathrm{t})$ are proportional to the gate signals that feed $\mathrm{Q}_{1-\mathrm{A}}$ and $\mathrm{Q}_{1-\mathrm{B}}$. Obviously, the gate signals that feed $\mathrm{Q}_{2-\mathrm{A}}$ and $\mathrm{Q}_{2-\mathrm{B}}$ are the complementary ones. Therefore, and as will be shown below, the remaining part of the control is focused systems on generating the gate signals

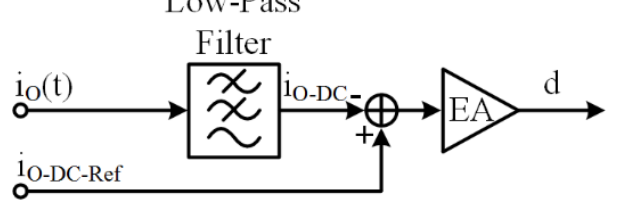

Fig. 11. Duty cycle calculator block

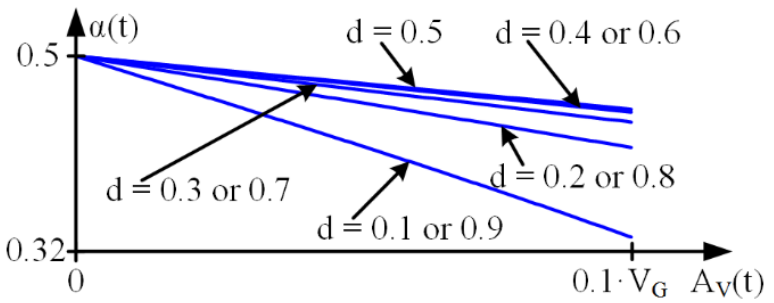

Fig. 12. $\alpha(t)$ versus $A_{V}(t)$ when $A_{V}(t)<<V_{G}$.

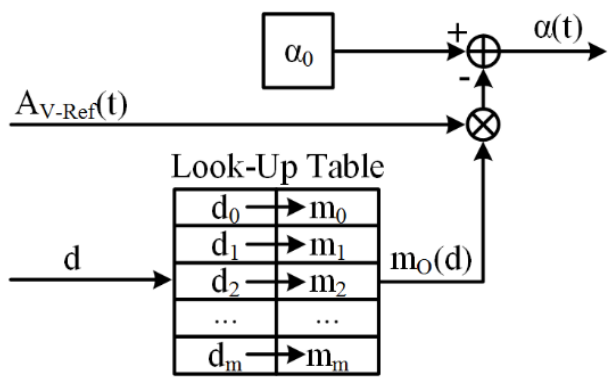

Fig. 13. $\alpha(\mathrm{t})$ calculator block.

that feed $\mathrm{Q}_{1-\mathrm{A}}$ and $\mathrm{Q}_{2-\mathrm{A}}$. The first step is to calculate the provisional rising and falling edges of the pulses. The reason that explains why they are provisional values is included in the next block. According to Section II.B, the provisional edges can be calculated following these equations:

$$
\begin{aligned}
& r_{e-A}(t)^{\prime}=\beta(t)-\frac{\alpha(t)}{2}-\frac{d(t)}{2}, \\
& f_{e-A}(t)^{\prime}=\beta(t)-\frac{\alpha(t)}{2}+\frac{d(t)}{2}, \\
& r_{e-B}(t)^{\prime}=\beta(t)+\frac{\alpha(t)}{2}-\frac{d(t)}{2}, \\
& f_{e-B}(t)^{\prime}=\beta(t)+\frac{\alpha(t)}{2}+\frac{d(t)}{2},
\end{aligned}
$$

where $\mathrm{r}_{\mathrm{e}-\mathrm{A}}(\mathrm{t})^{\prime}, \mathrm{f}_{\mathrm{e}-\mathrm{A}}(\mathrm{t})^{\prime}, \mathrm{r}_{\mathrm{e}-\mathrm{B}}(\mathrm{t})^{\prime}$, and $\mathrm{f}_{\mathrm{e}-\mathrm{B}}(\mathrm{t})^{\prime}$, are the provisional rising edge of the phase $\mathrm{A}$, the provisional falling edge of the phase $\mathrm{A}$, the provisional rising edge of the phase $\mathrm{B}$ and the provisional falling edge of the phase B, respectively.

\section{E. Definitive Edges Calculator}

The aforementioned rising and falling edges are provisional values because they are only valid if the pulse that 
must be generated does not exceed the limits of its switching period. Take into account that if a provisional edge is lower than 0 or higher than 1 , it means that the pulse exceeds its switching period. Fig. 14 shows an example of a pulse that tries to invade the previous switching period (this situation will be noted as Situation 2 in the following explanation). Considering the maximum and minimum values of $\alpha(\mathrm{t}), \beta(\mathrm{t})$ and $\mathrm{d}$, there are five possible situations:

Situation 1: $r_{e}(t)^{\prime}>0$ and $f_{e}(t)^{\prime}<1$. The pulse does not exceed its switching period. In this situation, the definitive rising and falling edges $\left[\mathrm{r}_{\mathrm{e}}(\mathrm{t})\right.$ and $\left.\mathrm{f}_{\mathrm{e}}(\mathrm{t})\right]$ are equal to the provisional ones.

Situation 2: $r_{e}(t)^{\prime}<0$ and $f_{e}(t)^{\prime}<1$. The pulse exceeds its switching period and a part of it tries to invade the previous one (see Fig. 14). In this situation, the part of the pulse that tries to appear in the previous switching period must be moved to the end of the correct one by adding " 1 " to $r_{e}(t)$ ".

Situation 3: $r_{e}(t)^{\prime}<1$ and $f_{e}(t)^{\prime}>1$. The pulse exceeds its switching period and a part of it tries to invade the next one. In this situation, the part of the pulse that tries to appear in the next switching period must be moved to the beginning of the correct one by subtracting " 1 " to $\mathrm{f}_{\mathrm{e}}(\mathrm{t})$ '.

Situation 4: $r_{e}(t)^{\prime}<0$ and $f_{e}(t)^{\prime}<0$. The pulse exceeds its switching period and tries to invade completely the previous one. In this situation, the pulse must be moved to the correct switching period by adding " 1 " to both $\mathrm{r}_{\mathrm{e}}(\mathrm{t})$ ' and $\mathrm{f}_{\mathrm{e}}(\mathrm{t})$ '.

Situation 5: $r_{e}(t)^{\prime}>1$ and $f_{e}(t)^{\prime}>1$. The pulse exceeds its switching period and tries to invade completely the next one. In this situation, the pulse must be moved to the correct switching period by subtracting " 1 " to both $\mathrm{r}_{\mathrm{e}}(\mathrm{t})$ ' and $\mathrm{f}_{\mathrm{e}}(\mathrm{t})$ '.

\section{F. Pulse-Width and Pulse-Phase Modulator (PWPPM)}

This block is responsible for generating the pulses of a buck phase by knowing their definite rising and falling edges. As in the case of PWM, the pulse generation is performed by making a comparison with a sawtooth signal whose frequency is $f_{\mathrm{SW}-\mathrm{RM}}$ and whose maximum and minimum values are 1 and 0 , respectively. Differently from PWM, the sawtooth signal is compared to two references: the definitive rising and falling edges (see Fig. 15). In addition, the output of the PWPPM depends on the aforementioned situations. When the situation is 1,4 or 5 ; $\mathrm{f}_{\mathrm{e}}(\mathrm{t})$ is higher than $\mathrm{r}_{\mathrm{e}}(\mathrm{t})$ and the output is:

$$
\begin{aligned}
& v(t)= \\
& =\left(\text { Sawtooth }>r_{e}(t)\right) \text { AND }\left(\text { Sawtooth }<f_{e}(t)\right) .
\end{aligned}
$$

On the other hand, when the situation is 2 or $3, \mathrm{f}_{\mathrm{e}}(\mathrm{t})$ is lower than $r_{e}(t)$ and the output must be:

$$
\begin{aligned}
& v(t)= \\
& =\left(\text { Sawtooth }>r_{e}(t)\right) \text { OR }\left(\text { Sawtooth }<f_{e}(t)\right) .
\end{aligned}
$$

Fig. 16 shows the scheme of the PWPPM block.

\section{EXPERIMENTAL RESULTS}

A two-phase synchronous buck converter with $10^{\text {th }}$ order Butterworth filter $\left(f_{C}=650 \mathrm{kHz}\right)$ was built in order to validate the proposed technique (see Fig. 17). This power converter supplies a string of five HB-LEDs (W42180 Seoul Semiconductor). The switching frequency is $500 \mathrm{kHz}$, the input voltage is $30 \mathrm{~V}$ and the maximum power is around 10 W. SSM3K361R MOSFETs are used driven by EL7156 ICs. Fig. 18 shows the main waveforms of the HB-LED driver.

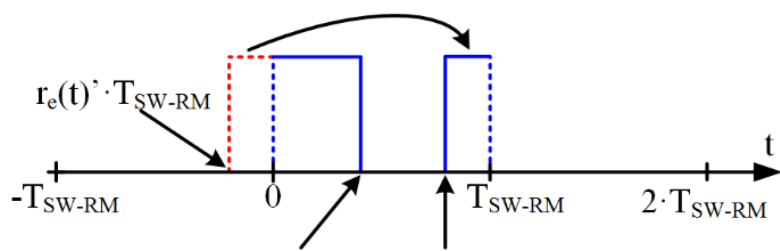

$\mathrm{f}_{\mathrm{e}}(\mathrm{t}) \cdot \mathrm{T}_{\mathrm{SW}-\mathrm{RM}} \quad \mathrm{r}_{\mathrm{e}}(\mathrm{t}) \cdot \mathrm{T}_{\mathrm{SW}-\mathrm{RM}}=\left[\mathrm{r}_{\mathrm{e}}(\mathrm{t})^{\prime}+1\right] \cdot \mathrm{T}_{\mathrm{SW}-\mathrm{RM}}$

Fig. 14. Example of a pulse that tries to invade the previous switching period

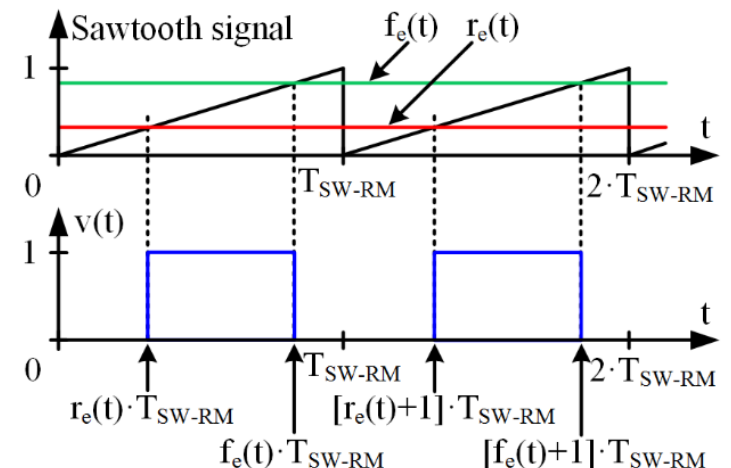

Fig. 15. Main waveforms of the PWPPM block.

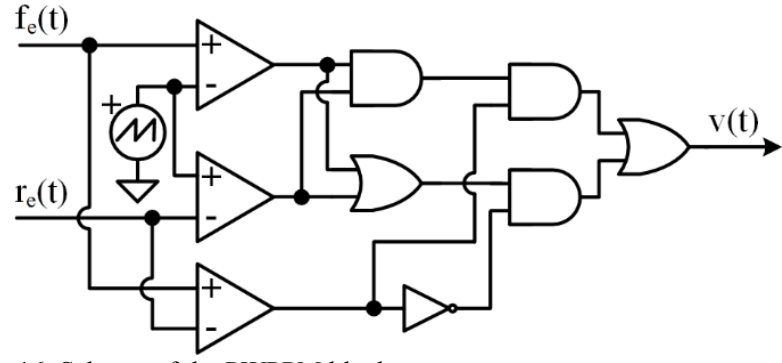

Fig. 16. Scheme of the PWPPM block.

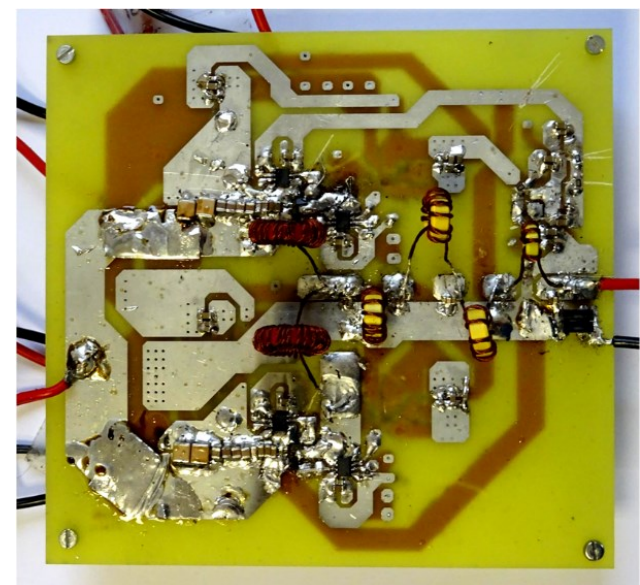

Fig. 17. Prototype of the HB-LED driver for VLC.

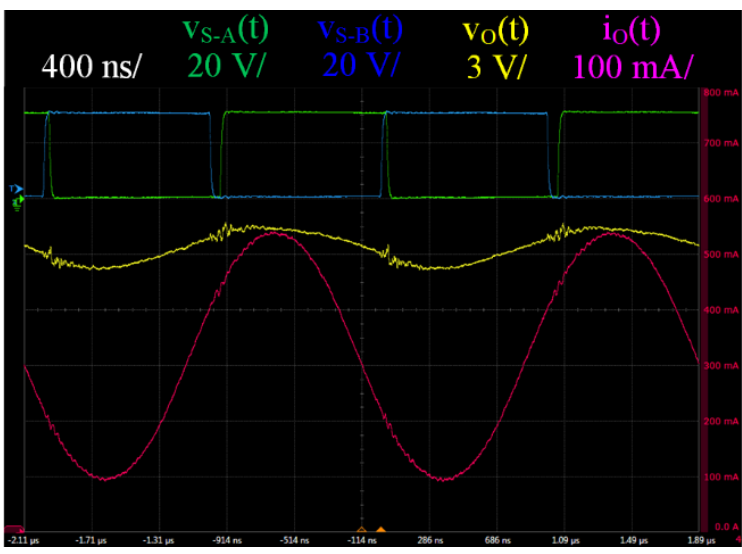

Fig. 18. Main experimental waveforms of the HB-LED driver for VLC 
In order to evaluate the communication capability, a signal that is made up of six carriers is reproduced. Their frequencies are $475 \mathrm{kHz}, 485 \mathrm{kHz}, 495 \mathrm{kHz}, 505 \mathrm{kHz}, 515 \mathrm{kHz}$ and 525 $\mathrm{kHz}$. A commercial receiver (PDA10A-EC) provides an output voltage $\left[\mathrm{V}_{\mathrm{RX}}(\mathrm{t})\right]$ proportional to the received light intensity. Fig. 19 shows the main waveforms of the VLC setup when the aforementioned signal is transmitted. The received signal is demodulated offline by using Matlab in order to obtain both the envelope of the received signal $\left[A_{R X}(t)\right]$ and its instantaneous phase $\left[\phi_{R X}(t)\right]$. This allows us to compare these components to the ideal ones $\left[\mathrm{A}_{\text {Ideal }}(\mathrm{t})\right.$ and $\phi_{\text {Ideal }}(\mathrm{t})$, respectively] (see Fig. 20).

Fig. 21 shows that the efficiency of the proposed power converter ranges between $95 \%$ and $96.5 \%$ depending on the dimming level. It must be pointed out that the efficiency is quite independent of $\mathrm{Av}_{\mathrm{V}}(\mathrm{t})$.

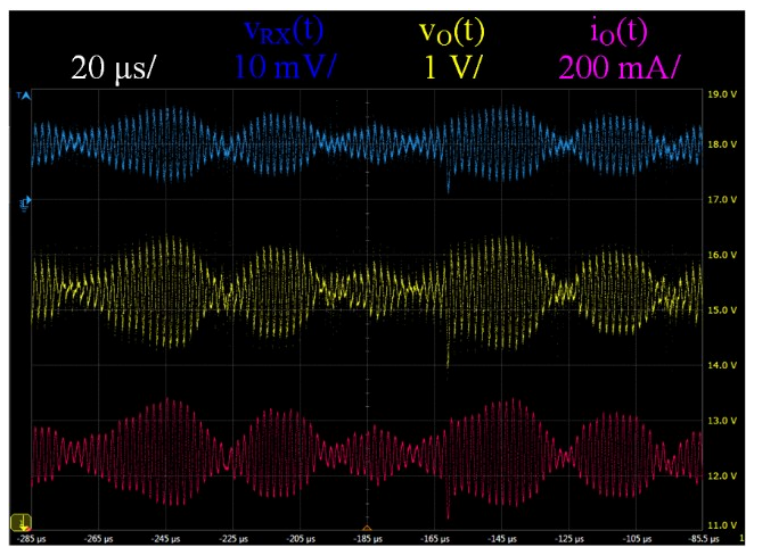

Fig. 19. Main experimental waveforms of the VLC link (distance: $40 \mathrm{~cm}$ ).

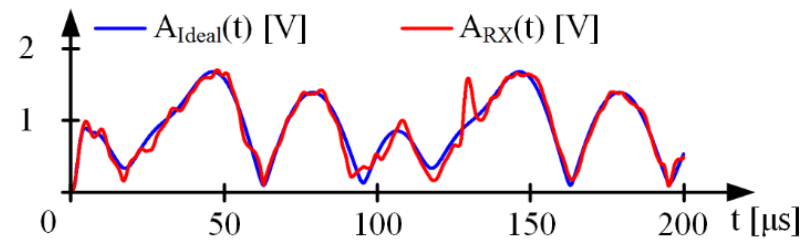

(a)

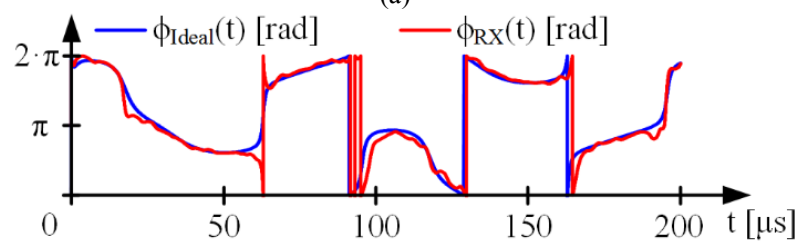

(b)

Fig. 20. Comparison between the ideal and the received envelope and instantaneous phase (link distance: $60 \mathrm{~cm}$ ). Note that the envelopes are normalized to have power equal to $1 \mathrm{~W}$ in order to enable the comparison.

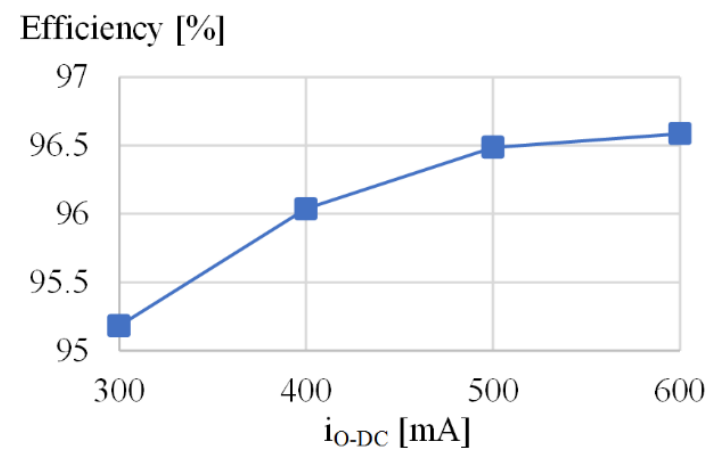

Fig. 21. Efficiency versus dimming level.

\section{CONCLUSIONS}

The RM technique provides interesting benefits to be applied in the HB-LED drivers of VLC transmitters. Since the power converter is responsible not only for the lighting (i.e., the biasing of the HB-LEDs), but also for the communication, it avoids the use of a LPA. As a consequence, the transmitter is simpler and more efficient. In comparison to other approaches based on the use of pulse-width modulated dc-dc power converters, it requires a much lower switching frequency. In this way, the impact of switching losses is mitigated and both the devices of the power stage and the digital system responsible for the gate signals generation are more affordable. The main drawback of the RM technique is that it has not been demonstrated that it is valid for reproducing MCM schemes, which are the preferred modulation schemes for VLC. This point is successfully addressed in this paper, validating the use of the RM technique for MCM schemes and studying in detail the control system of the power converter.

\section{ACKNOWLEDGMENT}

This work has been supported by the Spanish Government under the Project MINECO-17-DPI2016-75760-R, the scholarship FPU14/03268 and the Principality of Asturias under the Project FC-15-GRUPIN14-143, the Project SV-PA17-RIS3-4 and by European Regional Development Fund (ERDF) grants.

\section{REFERENCES}

[1] Cisco Systems, "Cisco visual networking index: global mobile data traffic forecast update, 2016-2021", Feb. 2017. [Online]. Available: http://www.cisco.com/c/en/us/solutions/collateral/serviceprovider/visual-networking-index-vni/mobile-white-paper-c11520862.html

[2] A. Jovicic, J. Li and T. Richardson, "Visible light communication: opportunities, challenges and the path to market," in IEEE Communications Magazine, vol. 51, no. 12, pp. 26-32, December 2013.

[3] A. Wilkins, J. Veitch and B. Lehman, "LED lighting flicker and potential health concerns: IEEE standard PAR1789 update," 2010 IEEE Energy Conversion Congress and Exposition, Atlanta, GA, pp. 171-178, 2010.

[4] J. Vučić, C. Kottke, K. Habel and K. D. Langer, "803 Mbit/s visible light WDM link based on DMT modulation of a single RGB LED luminary," Optical Fiber Communication Conference and Exposition (OFC/NFOEC), 2011 and the National Fiber Optic Engineers Conference, Los Angeles, CA, 2011, pp. 1-3.

[5] J. G. Proakis, Digital communications, 4th ed. New York: McGrawHill, 2000.

[6] J. Armstrong, "OFDM for optical communications," in Journal of Lightwave Technology, vol. 27, no. 3, pp. 189-204, Feb. 2009.

[7] J. Sebastián, D. G. Lamar, D. G. Aller, J. Rodríguez and P. F. Miaja, "On the role of power electronics in visible light communication," in IEEE Journal of Emerging and Selected Topics in Power Electronics.

[8] J. Rodríguez, D. G. Lamar, D. G. Aller, P. F. Miaja and J. Sebastián, "Efficient visible light communication transmitters based on switchingmode dc-dc converters," in Sensors, vol. 18, no. 4, 2018.

[9] J. Rodriguez, D. G. Lamar, P. Fernandez Miaja, D. G. Aller and J. Sebastian, "Power efficient VLC transmitter based on pulse-width modulated DC-DC converters and the split of the power," in IEEE Transactions on Power Electronics.

[10] V. Yousefzadeh, Narisi Wang, Z. Popovic and D. Maksimovic, "A digitally controlled DC/DC converter for an RF power amplifier," in IEEE Transactions on Power Electronics, vol. 21, no. 1, pp. 164-172, Jan. 2006.

[11] J. Rodriguez, P. Fernandez Miaja, D. G. Lamar and J. Sebastian, "Reproducing single-carrier digital modulation schemes for VLC by controlling the first switching harmonic of the DC-DC power converter output voltage ripple," in IEEE Transactions on Power Electronics. 